\title{
OPTIMIZACIÓN DE FUNCIONAMIENTO DE UNA BOMBA DE ARIETE*
}

\author{
Espinoza Montes, Ciro Abelardoํㅜ Lazo Baltasar, Brecio Daniel ${ }^{2}$ y Huari Vila, Oscar Paúl ${ }^{3}$ \\ Facultad de Ingeniería Mecánica de la Universidad Nacional del Centro del Perú
}

\begin{abstract}
RESUMEN
Teniendo como finalidad brindar tecnologías apropiadas para la utilización de agua para diversos fines en la producción agrícola y pecuaria en el Valle del Mantaro se ha formulado un sistema problemático que permita construir una bomba de ariete funcional y accesible a los productores. El propósito de la presente investigación es determinar la altura de caída y la altura de elevación para optimizar el caudal de elevación de agua en una bomba de ariete hidráulico. Para lo cual se construyó un prototipo construido con materiales sencillos, accesibles y compatibles con la cultura del productor del Valle del Mantaro. Este prototipo se sometió a manipulación experimental mediante un diseño factorial $2^{2}$, en riachuelos del distrito de San Agustin de Cajas de la provincia de Huancayo. Los resultados se midieron en la variable: caudal de elevación de agua, las variaciones se realizaron en las variables independientes: altura de caída de agua y altura de elevación de agua; desarrollando un modelo con tres réplicas y cuatro tratamientos. Como resultado, si la altura de caída de agua es mayor y la altura de elevación de agua es menor, se maximizará el caudal de elevación de agua.
\end{abstract}

Palabras claves: golpe de ariete, experimento, diseño factorial.

\section{OPTIMIZING THE PERFORMANCE OF THE BATTERING RAM PUMP}

\begin{abstract}
Having as main purpose to furnish adopted techmologies for the water usage for different matters in the agricultural and catlle production in the Mantaro Valley its been formulated as problematic system that letstus a pathering ram pump practical and accessible to the producers the main goal of this investigation is to determine the height of water and the height of elevation to optimize the elevation of the water volume in the hy droullic battering ram pump. For these means a prototype pump was constructed using simple accessible materials and compatible with the Mantaro Vallery producer. Culture this pump was put to an experimental manipulation by means of 2 factorialin design a brook in San Augustin de Cajas District, in the Province of Huancayo. The results were measured according to the following variables: The water elevation volume, the variations were given in the independent variables: heigth in the water filling, and height in the elevation of water; developing a model with three replies and four treatments: As a result, it was golten that, if the helght dropping water is higher and the height of water elevation is lower. The elevation of water volume will be maximized.
\end{abstract}

Key words: hydraulic ram, experiment, factorial design.

\section{INTRODUCCIÓN}

El presente trabajo de investigación tiene por objetivo principal determinar la altura de caída y la altura de elevación para optimizar el caudal de elevación de agua en una bomba de ariete hidráulico. Para lo cual se ha formulado el problema: ¿Cómo influye la variación de la altura de caída y la altura de elevación en el caudal de elevación de agua en una bomba de ariete hidráulico?, la hipótesis que guió la investigación fue: si deseamos elevar agua a una gran altura, debemos también maximizar la caída de agua para lograr un caudal óptimo en una bomba de ariete hidráulico. Las variables que nos permitieron realizar la experimentación son:

- $\quad \mathrm{Vd}=$ caudal de elevación de agua

- Vi1=altura de caída de agua

- $\quad$ Vi2= altura de elevación de agua

Este trabajo de investigación fue recibido el 20/03/2007 retornado para su revisión 20/06/2007 y aprobado para su publicación 10/12/2007

1. Email: ciroespinoza@hotmail.com

2. Email: baltazarlazo@hotmail.com

3. Email: oshu25@hotmail.com 
La bomba de Ariete materia del presente estudio es fabricada íntegramente con elementos de plástico, a excepción de las válvulas check y de pie que son de bronce. La metodología utilizada en la investigación es experimental, el diseño utilizado es el factorial $2^{2}$. Los resultados se midieron en la variable caudal de elevación de agua, las variaciones se realizaron en las variables independientes altura de caída de agua y altura de elevación de agua; desarrollando un modelo con tres réplicas y cuatro tratamientos.

\section{MATERIAL Y MÉTODOS}

\section{Material}

Los materiales utilizados para la investigación fueron:

- Bomba de ariete

- Módulo de prueba de bomba de ariete

- Medidores de caudal

- Flexómetro

Los materiales utilizados en la fabricación del prototipo de la bomba de ariete se encuentran en la tabla 1.

Tabla 1. Materiales para la fabricación del prototipo.

\begin{tabular}{|c|c|c|c|c|}
\hline $\mathrm{N}^{\circ}$ & Cant. & Descripción & $\begin{array}{c}\text { Precio } \\
\text { Unitario } \\
\end{array}$ & Importe \\
\hline 1 & 1 & $\begin{array}{l}\text { Acople para } \\
\text { manguera }\end{array}$ & Sl.0.50 & Sl. 1,00 \\
\hline 2 & 3 & Cinta de teflón & S/.1.00 & S/. 3,00 \\
\hline 3 & 3 & Codo de 1" & S/.1.00 & S/. 3,00 \\
\hline 4 & 1 & Llave de paso & $\mathrm{S} / .2 .50$ & SI. 2,50 \\
\hline 5 & 20 & Manguera & S/.1.00 & $S / .20,00$ \\
\hline 6 & 8 & Niples de 1" x 4" & S/.3.00 & $S / .24,00$ \\
\hline 7 & 1 & Niples de 1/2" x $3 "$ & S/.1.00 & S/. 1,00 \\
\hline 8 & 1 & Reductor de 1" a 1/2" & $\mathrm{S} / .2 .00$ & Sl. 1,00 \\
\hline 9 & 1 & Reductor de 1" a 3/4" & S/.3.00 & Sl. 1,00 \\
\hline 10 & 2 & T de 1" con rosca & S/.1.00 & S/. 2,00 \\
\hline 11 & 1 & Tubo de 1" & SI.16.00 & S/. 16,00 \\
\hline 12 & 1 & Unión 1" & Sl.1.00 & SI. 1,00 \\
\hline 13 & 1 & Válvula de pie(sim)de 1" & $S / 30,00$ & $S / 30,00$ \\
\hline 14 & 1 & Válvula check de 1" & $S / 30,00$ & $\mathrm{~S} / .30,00$ \\
\hline \multirow[t]{2}{*}{15} & 1 & $\begin{array}{l}\text { Válvula de } \\
\text { compuerta }\end{array}$ & $S / 14,00$ & $S / .14,00$ \\
\hline & & TOTAL & Sl. & 149,50 \\
\hline
\end{tabular}

\section{Métodos}

El tipo de investigación es aplicada y se utilizó el método de investigación experimental.

El diseño es experimental y se utilizó el diseño factorial $2^{2}$.
Las variables: altura de caída del agua $\mathrm{A}=\mathrm{h}$ y la altura de elevación $B=H$ con dos niveles cada una.

Para validar la hipótesis se utilizó la siguiente hipótesis estadística:

$\mathrm{H}_{0}: \mu_{1}=\mu_{2}$ (medias entre tratamientos)

$\mathrm{H}_{1}: \mu_{1} \neq \mu_{2}$

\section{Prototipo}

\section{Componentes de un sistema de ariete:}

En la figura 1 se observa los componentes del sistema:

h: Fuente de alimentación ubicada a una altura

Q: Tubería de impulso, lleva un caudal

A: Válvula de impulso

B: Válvula de retención

P: Cámara de aire

q: Tubería de descarga, lleva un caudal

$\mathrm{H}$ : Tanque de abastecimiento, donde se realiza la descarga a una altura.

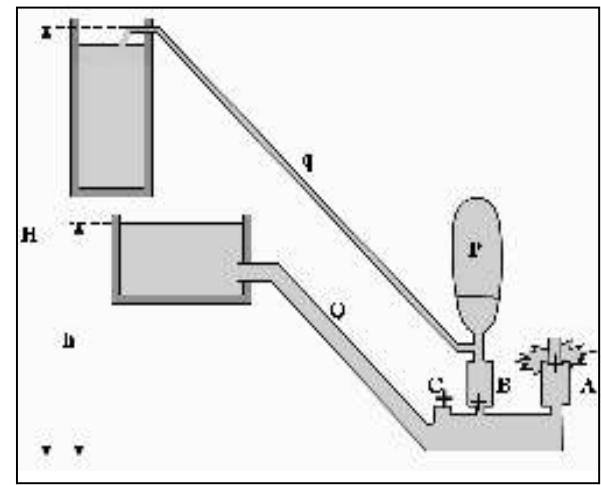

Figura 1. Esquema de instalación y funcionamiento del ariete hidráulico.

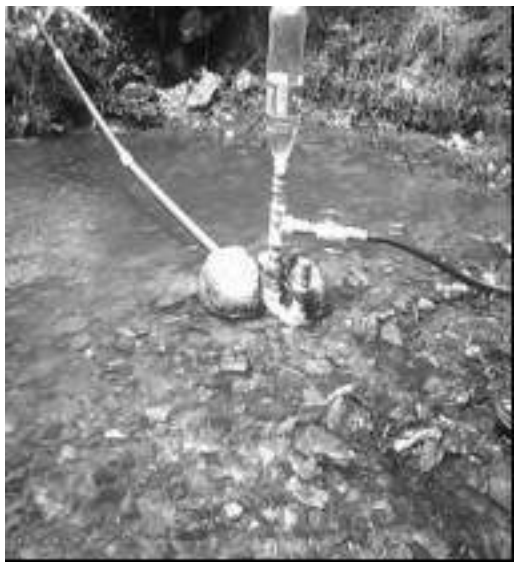

Figura 2. Prototipo en funcionamiento. 


\section{Analogía entre un transformador eléctrico y un sistema de ariete}

El transformador eléctrico recibe una tensión baja (en voltios) con una corriente eléctrica (en amperes) relativamente alta, y lo transforma en un régimen de mayor tensión y menor intensidad de corriente.

Análogamente ocurre a nivel hidráulico en una instalación con un ariete. El aparato recibe el gran caudal con la carga moderada o baja y lo transforma en un régimen de mayor presión con un menor caudal.

\section{RESULTADOS}

El promedio de bombeo con una caída de 1,5 metros a una altura de 8 metros es de 1,6 m3/día, por lo que con el presente estudio, lograremos conocer con exactitud el comportamiento real de las variables y optimizar el funcionamiento de la bomba para lograr el mejor caudal.

$\mathrm{A}=$ altura de caída $1,5 \mathrm{~m}$ y $1,75 \mathrm{~m}$

$B=$ altura de elevación $8 \mathrm{~m}$ y $10 \mathrm{~m}$.

\section{Análisis factorial}

Para realizar el análisis factorial se ha tomado cuatro representaciones y tres réplicas, como aparecen en la tabla 2.

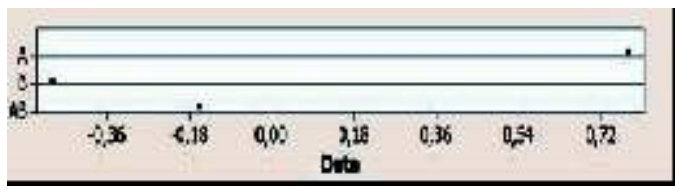

Figura 3. Posicionamiento de las variables A y B

En la figura 3 se observa gráficamente el posicionamiento de estos efectos.

\section{Análisis de varianza para los datos obtenidos}

El análisis de varianza para los datos obtenidos aparecen en la tabla 2.

SSt tiene 4(3)-1 grados de libertad $=11$

Tabla 2. Tratamientos y réplicas

\begin{tabular}{cccccc}
\hline $\begin{array}{l}\text { Repre- } \\
\text { senta }\end{array}$ & $\begin{array}{c}\text { Combinación } \\
\text { de } \\
\text { tratamientos }\end{array}$ & I & II & III & Total \\
\hline 1 A baja, B baja & 0,57526 & 0,55824 & 0,56106 & 1,69456 \\
a A alta, B baja & 1,39534 & 1,80234 & 1,26849 & 4,46617 \\
b A baja, B alta & 0,24007 & 0,20844 & 0,25983 & 0,70834 \\
ab A alta, B alta & 0,99734 & 0,79124 & 0,78053 & 2,569 \\
\hline
\end{tabular}

Se tiene 4(3-1) grados de libertad $=8$

Tabla 3. Tratamientos y réplicas

Del teorema de Cochran: A Fo>11,3

B Fo $>11,3$

Por lo tanto la hipótesis nula es falsa.

\section{DISCUSIÓN}

De las estimaciones realizadas obtenemos los siguientes valores para cada uno de los datos (tabla 4):

$$
\begin{aligned}
& \text { DONDE: } \\
& A=\text { altura de toma } \\
& B=\text { altura de salida } \\
& \text { De la tabla anterior, las estimaciones de los efectos } \\
& \text { promedio son: }
\end{aligned}
$$

\begin{tabular}{ll}
$A=$ & 0,77206 \\
$B=$ & $-0,48055$ \\
$A B=$ & $-0,15181$ \\
\hline
\end{tabular}

Tabla 4. Resultado de estimaciones

\begin{tabular}{lll} 
Altura de caída de agua & $A$ & $=0,77206$ \\
Altura de elevación de agua & $B$ & $=-0,48055$ \\
Interrelación de ambas variables & $A B$ & $=-0,15181$ \\
\hline
\end{tabular}

Si deseamos maximizar la variable respuesta, entonces:

La variable A debe estar en su nivel máximo (debido a haber obtenido 0,77206)y

La variable $B$ debe estar en su nivel mínimo (debido a haber obtenido $-0,48055$ )

Estos resultados se corroboran con los obtenidos en el análisis de la varianza, por el método de Cochran, también determinamos que la hipótesis alterna $\left(\mathrm{H}_{1}\right)$ es verdadera.

\section{CONCLUSIONES}

Por tanto: Si deseamos elevar agua a una gran altura ( $\mathrm{H}$ en la figura 1), debemos también maximizar la caída de agua (h en la figura 1) para lograr un caudal óptimo en una bomba de ariete hidráulico.

\begin{tabular}{ccccc}
\hline $\begin{array}{c}\text { Fuente } \\
\text { de } \\
\text { variación }\end{array}$ & $\begin{array}{c}\text { Suma } \\
\text { de } \\
\text { cuadrados }\end{array}$ & $\begin{array}{c}\text { Grado } \\
\text { de } \\
\text { libertad }\end{array}$ & $\begin{array}{c}\text { Media } \\
\text { de } \\
\text { Cuadrados }\end{array}$ & Fo \\
\hline$A$ & 1,78825 & 1 & 1,78825 & 76,52084 \\
$B$ & 0,69278 & 1 & 0,69278 & 29,64456 \\
$A B$ & 0,06914 & 1 & 0,06914 & 2,95839 \\
Error & 0,18696 & 8 & 0,02337 & \\
Total & 2,73711 & 11 & & \\
& De tablas & 11,3 & & \\
\hline
\end{tabular}




\section{LITERATURA CITADA}

Arancibia, M. G., 2005. Bombeo de agua sin costo, fecha de acceso 25 de marzo de 2005, disponible en http://www.uvm.cl/congresoenergia/doc/p6.pdf

Cervantes, M. E. 1976. Teoría de las bombas centrífugas y algunas de sus aplicaciones. Tesis Profesional. ENA. Chapingo México.

Del Valle, F. H. 1983. Fundamentos de electricidad para riego y agroindustrias. $\mathrm{UACH}$.
Karassik, I. J. 1984. Engineers guide centrifugal pumps. Mac Graw Hill Book Company, Inc. New York.

Montesinos, A. 1998. El ariete hidráulico, Centro Internacional de TecnologíaAgrícola.

Salvador, B. y Sánchez, C. P. 2005. Energías renovables. Fecha de acceso 25 de marzo de 2005, disponible en http://www.concytec.gob.pe/redandina/pnuma/reunregionales/2rcrdesk/23\%20casose xitosos/solartec.pdf 